\title{
Classification of Large Biometric Data in Database System
}

\author{
Varisha Alam, Mohammad Arif
}

\begin{abstract}
Biometrics" is got from the Greek word 'life' and 'measure' which implies living and evaluation take apart. It simply converts into "life estimation". Biometrics uses computerized acknowledgment of people, dependent on their social and natural attributes. Biometric character are data separated from biometric tests, which can use for examination with a biometric orientation. Biometrics involves techniques to unusually recognize people dependent on at least one inherent physical or behavior attribute. In software engineering, specifically, biometric is used as a form of character retrieve the Committee and retrieve command. Biometric identically utilized to recognize people in bunches that are in observation. Biometric has quickly risen like a auspicious innovation for validation and has effectively discovered a spot in most of the scientific safety regions. An effective bunching method suggest for dividing enormous biometrics data set through recognizable proof. This method depends on the changed $\mathrm{B}+$ tree is decreasing the discs get to. It diminishes the information recovery time and also possible error rates. Hence, for bigger applications, the need to reduce the data set to a more adequate portion emerges to accomplish both higher paces and further developed precision. The main motivation behind ordering is to recover a small data set for looking through the inquiry.
\end{abstract}

Keywords: Data Structure, Neural Network and Fuzzy Logic.

\section{INTRODUCTION}

"Biometric" means "living calculation" however, the term is typically related to the utilization of distinctive activity features to identify a particular. However, biometric recognition usually encompasses a rich wide application as computer interface becomes a lot essential. Significant, the individual with whom you are talking is a significant piece of human methods. The strategy of acknowledgment uphold biometric features these days ideal above conventional passwords and PIN-based strategies for different motive like the individual to be perceived is needed to be there at the hour of-acknowledgment. Biometric used "something you are" to confirm recognition. This may accept fingerprint, iris, hand calculation, vein design, voice secret key, or mark elements. Biometric can be utilized with a smart chip card to guarantee the client.

Manuscript received on February 20, 2021.

Revised Manuscript received on August 05, 2021.

Manuscript published on August 30, 2021.

* Correspondence Author

Varisha Alam*, Pursuing, MTech, Department of Computer Science and Engineering, Integral University, Lucknow, India.

Dr. Mohammad Arif, Associate Professor, Department of Computer Science \& Engineering, Integral University, Lucknow, India.

(c) The Authors. Published by Blue Eyes Intelligence Engineering and Sciences Publication (BEIESP). This is an open access article under the CC BY-NC-ND license (http://creativecommons.org/licenses/by-nc-nd/4.0/)
The client's biometric details is put away on a smart chip card, the savvy card is set in a per-user and a biometrics scanner per-uses the details to coordinate with it fraud of that on the smart chip card \{is often this will be . This is a quick, exact, and very secured sort of user authorization. A person's features can be utilized for biometrics can be recognized as far as the associated factors:

- Absoluteness- indicate that each individual must have the feature.

- Singleness - means how well the biometric split one person from another

- Collectability - refers to ease of gaining for dimension.

- $\quad$ Presentation - manages precision, speed, and fortitude of innovation utilized.

- Correctness- is level of agreement of a.

- Condition-is the facility of use of a alternative.

- Estimable- the asset must be appropriate for captured without waiting time and should be simple together the quality data inactively.

- Privacy - the procedure mustn't breach the privacy of the individual.

- Inimitable - the characteristics should be unreproducible by different way.

\section{VERIFICATION AND IDENTIFICATION}

A balanced partition of a represent biometric with putaway figures to check that the isolates that the person who she proclaims to be. A check will be worn out simultaneousness by a keen card, login name, or ID digit (because of "what she has" or "what she review"). The distinguishing proof exclusively prevails to perceive the difference if the separation of the biometrics chose to guidance in the information base comes inside a prior point entry(based on "what her identity is"). The strategy contains for the most part three stages: Information Acquisition Feature Extraction Coordinating, However, this entire interaction is not that simple. There are not many provocations that are looked at by any biometric validation framework. Throughout ID the framework must deal with an immense record and along these lines, time taken by it to announce a character mustn't be a lot. To serve its thought process, an ID framework wants a methodical looking and coordinating with calculation. The amount of inaccurate positive in the framework must not be exceptionally enormous as the components of the information base extend.

Published By:

Blue Eyes Intelligence Engineering and Sciences Publication

(C) Copyright: All rights reserved. 
A biometric framework is an example identification framework that works in an additional manner: Obtain biometric information from isolates, dynamic qualities set from the acquired data, and separate these attributes set against the guidance set in the data set. Biometrics generally execute embrace unique mark, face, iris, voice, mark, and hand calculation.

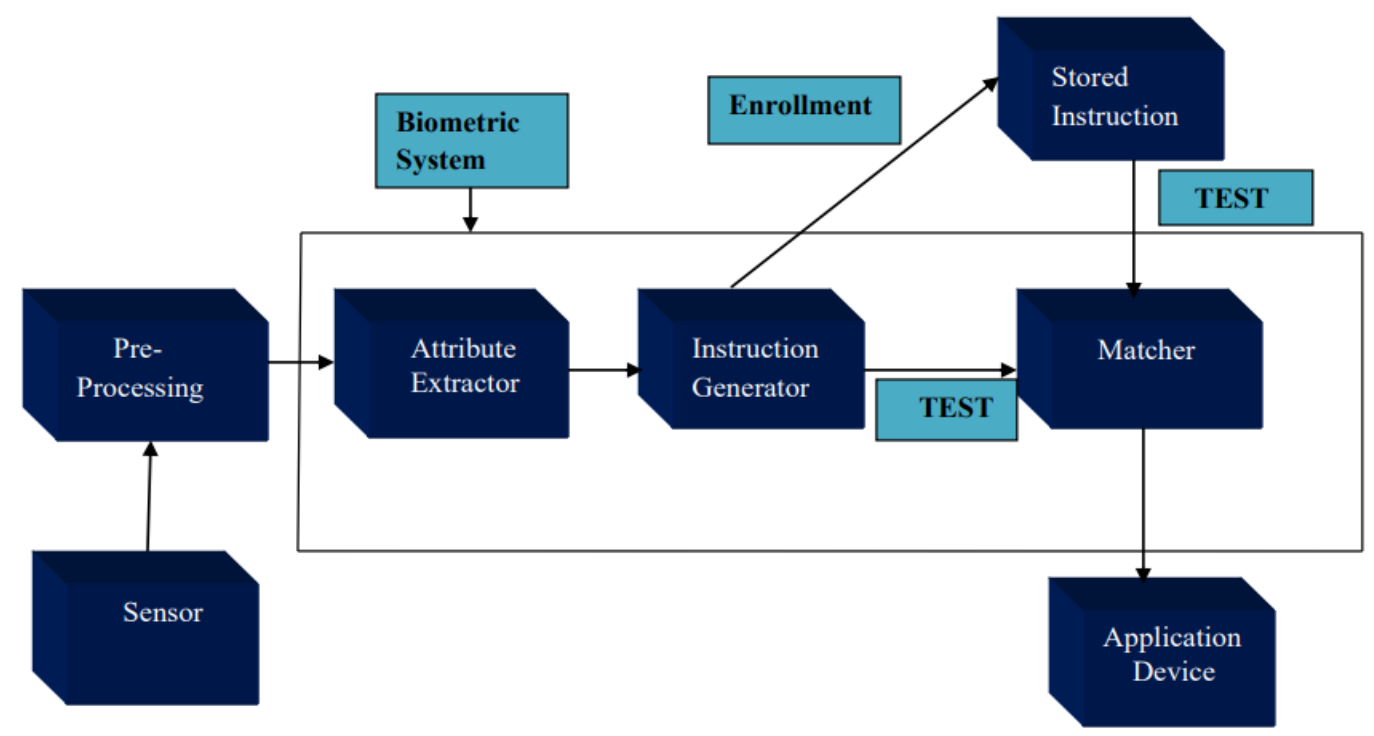

Figure 1. Generic Biometric System

\section{TYPES OF BIOMETRIC SYSTEM}

There are two sorts of biometrics:

3.1. Behavioral biometrics

3.2. Physical biometrics

\section{A. Behavioral biometrics}

It typically gauges the highlights which are gotten normally throughout a period. It is by and large utilized for a check.

Instances of social biometrics contain:

\section{B. Voice}

It proposes investigating vocal conduct. It is practically difficult to duplicate anybody's voice absolutely. Voice ID frameworks can recognize two very much like voices, including twins. Voice is an element of a person. Nonetheless, it isn't required to be suitably remarkable to approve acknowledgment of a person from an immense data set of detail.

\section{Signature}

It manages to investigate mark elements. Impression are a social biometrics that believers throughout a measure of your instance and are contrived by physical besides as passionate states of the signatories. The way during individual signs their name could be a trademark highlight of that careful individual.

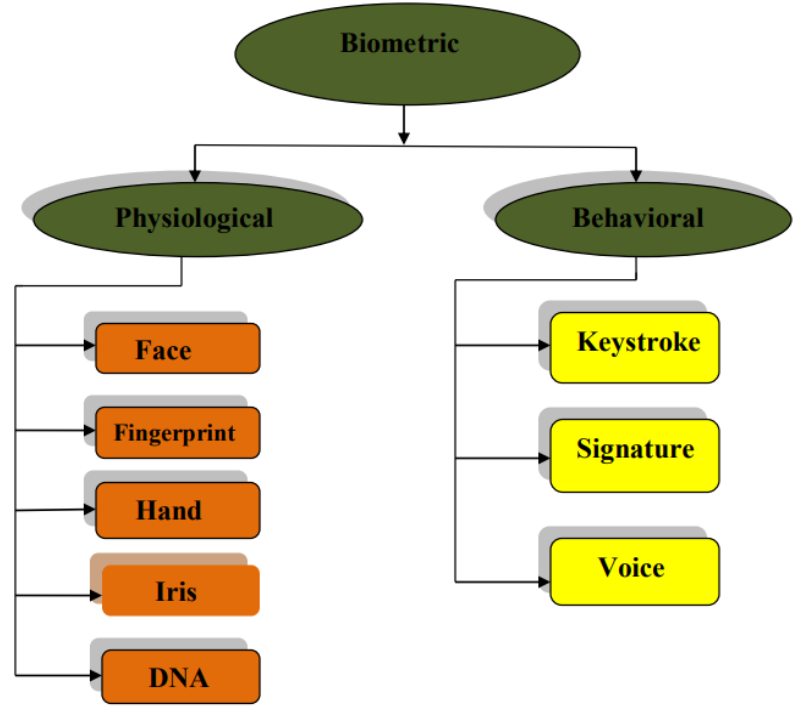

Figure 2. A Classification of Biometric Traits

\section{PERFORMANCE STANDARD}

For assessing the viability of a biometric framework, the resulting boundaries are utilized:

- False Acceptance Rate(FAR)

- False Rejection Rate (FRR)

- Genuine Accept Rate (GAR)

\section{A. False Acceptance Rate (FAR)}

This is a rate of recognition case in which uncertified individuals are inaccurately received. Like FAR, FRR is moreover a non-statically logical amount. This is frequently authorized to as close to FRR.

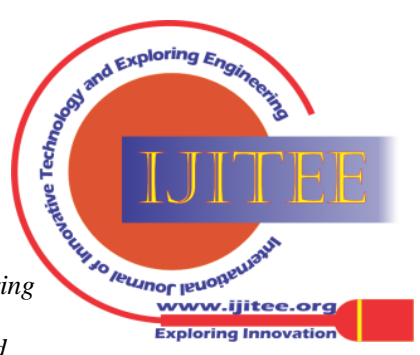




\section{B. False Rejection Rate (FRR)}

It is the number of times a biometric technique neglects to allow an uncertified individual. In applied science terms, FRR is type 1error. So much is furthermore called False Non-Match Rate (FNMR)

\section{Genuine Accept Rate (GAR)}

It is used to compute the reliability of the biometric process. A higher worth of GAR shows better execution.

\section{LITERATURE REVIEWS OF VARIOUS AUTHORS}

A. Iris Recognition Techniques(2015) Alice Nithya, C.Lakshmi:

Information of the associate degree life structures of an iris, a nearby history of iris has been started to be treated as a biometric asset and an overall structure of iris recognition system that is used. The principal objective of this work is to supply a timetable perspective on grouped iris acknowledgement strategies. Most of the works carried on iris acknowledgement is more allocate or less comparative anyway the prime centre was mainly made into four significant regions explicitly iris division, standardization which joins commotion evacuation, highlight extraction and characterization of iris layouts. Overtime 1993 - 2002, analysts were obsessed with creating calculations for every one of the four significant regions and were interested in fostering their frameworks. All through 2003 - 2009, significant exploration works had occurred on division stage which includes fragmenting the iris and diminishing the commotions present in it. Throughout 2007-2012, thought about the examination works were done on growing new element extraction calculations and grouping of iris further.

B. Fingerprint Quality Assessment and Its Evaluation(2016) Zhigang Yao, Jean-Marie Le Bars, Christophe Charrier, Christophe Rosen- berger

The evaluation system is in a situation to supply a detail live to exhibit what amount the normal metric adds to the advancement of the overall demonstration. By making attempts in these two features, we aim to noticed that few inquiries need to be replied to or consider extra evaluation:

1) Are those unique mark quality measurements dependent on multi attributes amazingly ready to make the mixed measurement relating?

2)To incorporate a typical outcome, it is important to examine whether learning earlier information on coordinating with execution love GMS is moderate or not?

This is frequently not to guarantee that quality isn't prescient to the coordinating with execution anyway one should take note of this constraint as existing coordinating with approaches are bad or strong \{to all/to all or partner degree/to any or all $\}$ picture settings, albeit picture goals are similarly on the edge to one Also, it's rationalist that anyhow 2 examples generate moderate performer achieved after they are of inferior standards., it is questionable moreover for the genuine coordinating with a score between two real examples if one among them has an abrupt quality.

C. Fingerprint Level one and Level two Attribute Enhancement to Improve Quality of Image.(2017) Sareeramana Aithal, Krishna Prasad Karani
Fingerprint picture improvement is one of the critical strides in the Mechanical Fingerprint Identification method. The unique fingerprint affirmation system execution constantly relies upon the nature of the fingerprint input picture. Individual fingerprints picture improvement is done by up the normal of ridge design or expanding the consistency of edge direction, which precisely implies the level 1 component, is uncovered and broke down for development reasons.

D. Fingerprint Database Indexing Methods(2019) Joannes Falade, Sandra Cremer, Christophe Rosenberger

A nearby investigation of four fingerprint ordering methodologies. Essential, certainly, factor and MCC approach. The ordering approaches under an overall investigation convention misuse FVC information bases and commonly expected an inconvenient huge data set for observing ordering techniques. The ordering strategies upheld course approach relies upon the 1-1 coordinating with calculations and thus the component of unique finger impression data sets.

E. An Investigation of Biometric Authentication in the Healthcare Environment(2020) Joannes Falade, Sandra Cremer, Christophe Rosenberger

A biometric framework for the medical care environment. The related approach in distinctive patients, both new and enlisted, in medical services data frameworks. This kind of biometric confirmation is moreover horrible material for work area/PCs, and tablets. The getting the periocular biometrics of each tolerant with their data and assent. The periocular space is that the area of the face that include the eyes, eyelids, eyelashes, eyebrows, and irises. Periocular biometrics are unmistakable to each person. The connecting a person's biometric information with their EMPI will be that the image utilized in care data framework to discover electronic medical services records. The EMPI is select for each persistent.

\section{ORDERING OF FINGERPRINT RECOGNITION SYSTEM}

Fingerprint acknowledgdement or fingerprint verification converse with an self-operating technique of collateral a test among 2 individuals fingerprints. Fingerprint are various kinds of biometric accustomed to establish a private and validate their uniqueness. As a result of their individuality and consistency above time, fingerprints are used for over an ages. Recently, turn into automated due to improvement in computing potentials.

\section{A. What is a fingerprint?}

The membrane on individual's fingertips have ridge and valley that along with form unique patterns. These shapes are grow underneath gestation and are lasting during the whole life span. Prints of these shapes are referred to as fingerprints. Wound like cuts, burns, bruises will briefly harm the quality of fingerprints however once fully well patterns are going to be repaired.

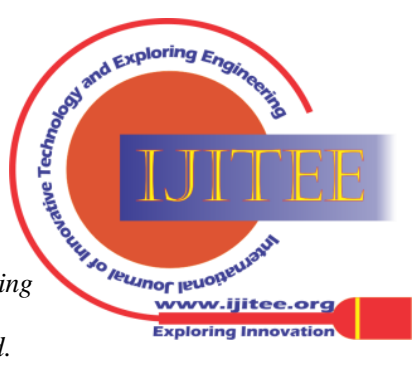


Through different observation it's been discover that no 2 persons have the equivalent fingerprints thus they are distinctive for each sole.

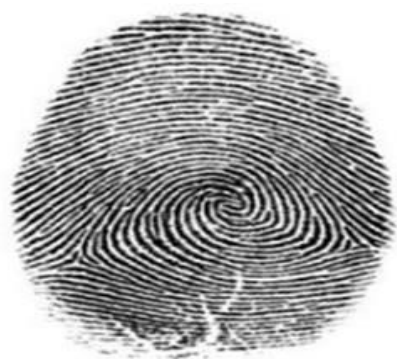

Fig. 3. Fingerprint Picture

But, by some serious analysis on fingerprint detection, fingerprints don't seem to be distinguished by their ridges and furrows, however by options referred to as Minutia, that are same irregular spots on their ridges. Between the variability of item varieties detailed on literature, two are mainly and insignificant usage:

- Ridges finishing, the quick ending of the ridge

- Ridge bifurcation- one ridge that split into two ridges
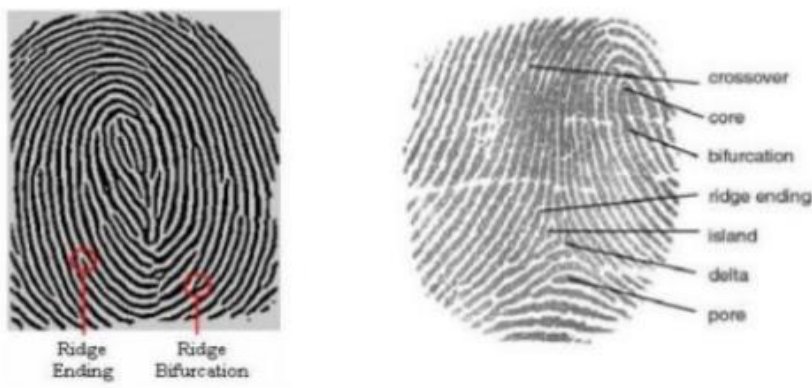

Fig.4. Two Fingerprints Details

Unique mark acknowledgment (here and there referenced as dactyloscopy) is that the strategy for figuring scrutinized an incredible finger impression against another finger not set in stone if the impressions are from indistinguishable fingers or palms. It accepts a couple of sub-areas one unique mark confirmation likewise the different finger impression recognizable proof. Moreover, not the same as the actual methodology for unique mark ID by a trained professional, the finger impression distinguishing proof here is alluded to as AFRS (Automatic Fingerprint Recognition System) that is programs based. In any case, through and through unique mark acknowledgment issues, either check (one to at least one coordinating) or ID (one to 1 many), the underlining standards of all around made public delineation of a finger impression and coordinating with stays as before.

\section{B. Multimodal biometric employment and authentication stage}

This paper consists of 2 sections named multimodal biometric employment phase and authentication phase. The multimodal biometric employment phase consists of the subsequent steps: the primary stage is to reinforce the key image of fingerprint and FKP by bar graph effort technique simultaneously. A consecutive step is to take out the feature and essential aims from the improved fingerprint and FKP image.
- Once extracting, the feature and essential points from fingerprint and FKP are cluster victimisation K-Means cluster algorithmic rule.

- The centre of mass standards calculated from the KMeans clustering algorithm are reborn into 128 bits of binary values from every biometric.

- The two 128 bits of fingerprint and FKP are concatenated as 256 combine bits and hold on within the database.

A consecutive section is the multimodal identification phase. The multimodal biometric verification consists of the subsequent steps:

- The method of generating 256 fused bits from fingerprint and FKP victimisation K-Means cluster algorithmic rule is that is same as the enrolment process.

- Consecutive step is to match the question 256 bits with the stored bits.

- The matching process is completed by XOR operation. If each of the bits are the same, XOR provides a worth of ' 0 ' or, both the bits are different, XOR gives value as ' 1 '.

- The last step is to decide that if all the 256 bits are zero, the user is genuine, else the user is a fraud.

Examinations during this paper are lead exploitation of the unique mark data FVC 2004. This information base comprises 4 sub-datasets, the essential three sub-datasets gathered from different sensors, and furthermore, the fourth sub-datasets created artificially. Each sub-information base contains eighty finger impression pictures. This information base comprises FKP pictures with its area of interest alone by trimming the external surface picture. This information base comprises 4 sub-datasets, they are left record FKP, left centre FKP, right list FKP, and right-centre FKP. Each sub-information base comprises a hundred 65 fingers of twelve pictures each. The information base comprises of660 organizers of 7920 FKP pictures.

\begin{tabular}{|l|l|l|l|}
$\begin{array}{l}\text { Name of } \\
\text { database }\end{array}$ & $\begin{array}{l}\text { Sensor } \\
\text { type }\end{array}$ & Picture size & Resolution \\
\hline DB1 & $\begin{array}{l}\text { Optical } \\
\text { sensor }\end{array}$ & $640 * 480$ & $500 \mathrm{dpi}$ \\
\hline DB2 & $\begin{array}{l}\text { Optical } \\
\text { detector }\end{array}$ & $328 * 364$ & $500 \mathrm{dpi}$ \\
\hline DB3 & $\begin{array}{l}\text { Thermal } \\
\text { extensive }\end{array}$ & & \\
\hline & detector & $300 * 480$ & 512 dpi \\
\hline------ & ----- & ------ & ------ \\
\hline DB4 & $\begin{array}{l}\text { SFinGe } \\
\text { v3.0 }\end{array}$ & $288 * 384$ & about 500 dpi \\
\hline
\end{tabular}

Fingerprint picture is increasing among bar graph coordination. The FKP image is additionally enhanced with histogram equalization. The feature and key point's extraction of fingerprint and FKP. Simulation is performed by ten pictures of every information set for each biometrics. For example, enrollment and also the verification part is taken from both biometric pictures of unique mark and FKP. Comprises of all boundaries that are used in this paper to play out every one of the means.

Published By:

Blue Eyes Intelligence Engineering

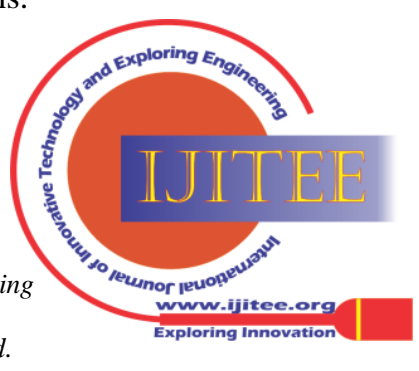



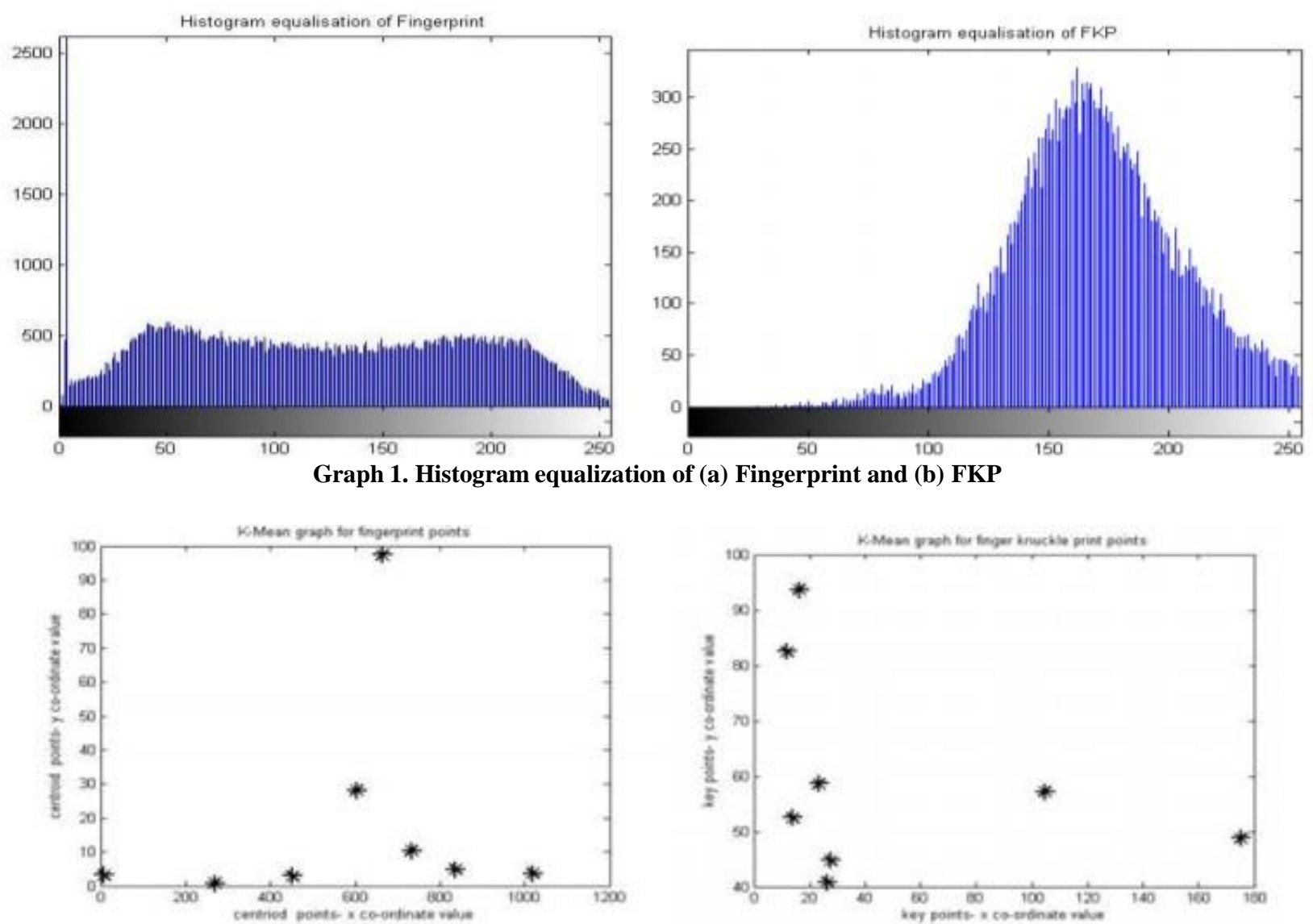

Graph 2.K-Mean clustering graph for (a) Fingerprint points and (b) FKP point

Table 1.Parameters used for multimodal biometric employment and authentication stage using fingerprint and

FKP

\begin{tabular}{|c|c|}
\hline \multicolumn{2}{|c|}{ Parameter } \\
\hline No. of DWT level (Finger print) & 2 level Haar decomposition \\
\hline No. of input spot (FKP) & $430-545$ spot \\
\hline K-Means clustering & 8 clusters \\
\hline Processing layout & Hex Decimal, Binary \\
\hline Fingerprint and FKP values & 256 bits(128+128) \\
\hline XOR comparison values & 256 bits \\
\hline
\end{tabular}

Consider as SV (saved value) and QV (query value) on the facts are to be equated. The MV (matched value) is the turnout of the XOR process of 2 completely dissimilar values. The MV is given by,

$$
i=1
$$

$$
\mathrm{MV}=\sum 256 \text { XOR }(, Q)
$$

in which one to 256 is the whole amount of amalgamated bits. If 2 qualities are autonomous, the MV among them must be equivalent to one, as independence suggests that the two sequences are entirely different. If the two principles are the same bits that processed either zero or one, then their XOR standard must be zero. The experiments are conducted discrimination fingerprint and FKP database.

\begin{tabular}{|c|c|}
\hline Process & Time(in Seconds) \\
\hline Fingerprint feature values generation & 3.20 \\
\hline FKP values generation & 2.30 \\
\hline Fused values & 0.53 \\
\hline Matching values & 0.32 \\
\hline
\end{tabular}

Table 3. Execution time for employment and authentication stage

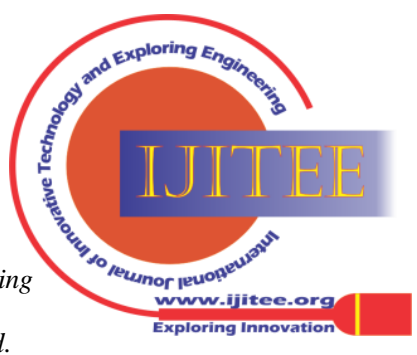




\section{PROPOSED METHODOLOGY}

Some techniques describe the accuracy of the biometric systems. The primary technique is to check the accuracy of the system and this might be done by several iterations utilized the K-Means clustering algorithm. If the number of iterations of the K-Means cluster rule increases, then the accuracy of matching SV with QV is additionally increased. The optimum centre of mass value is achieved if the number of iterations increases. If the optimum centre of mass worth is reached, it is inherent again into 128 binary bits with optimum value, so the accuracy of the recognition rate becomes immensely high.

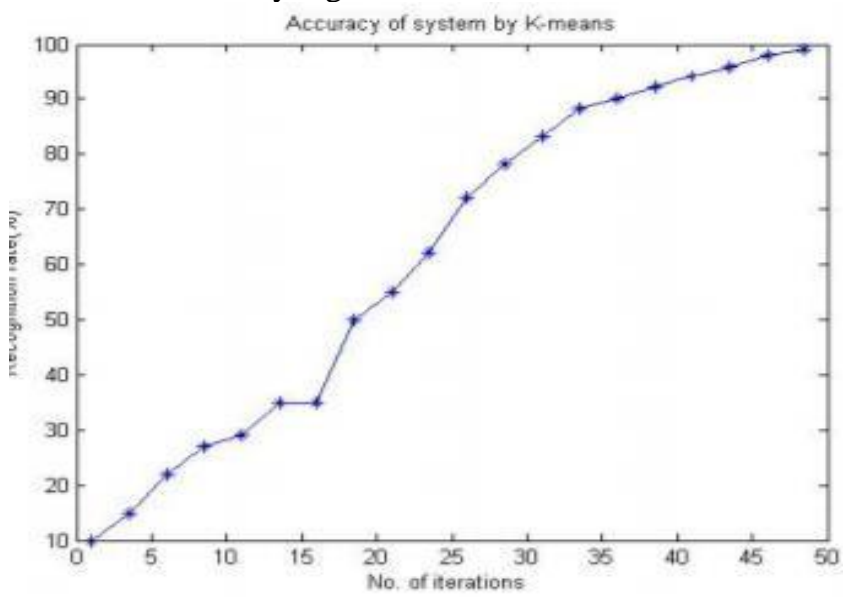

Graph 3.Precision of system by K-means algorithm

The second methodology is to judge the G-S curve with the accuracy of Genuine Acceptance Rate (GAR) with several bits (security) used within the system. This curve is obtained by varying the number of clusters victimisation KMeans cluster algorithmic program and by using the centre of mass bit conversion employed in this system. It is determined that the reliability of the system is acquired with $99.5 \%$ of GAR at 128 bits. The reliability of the method will increase

through the increasing digit of bits generated.

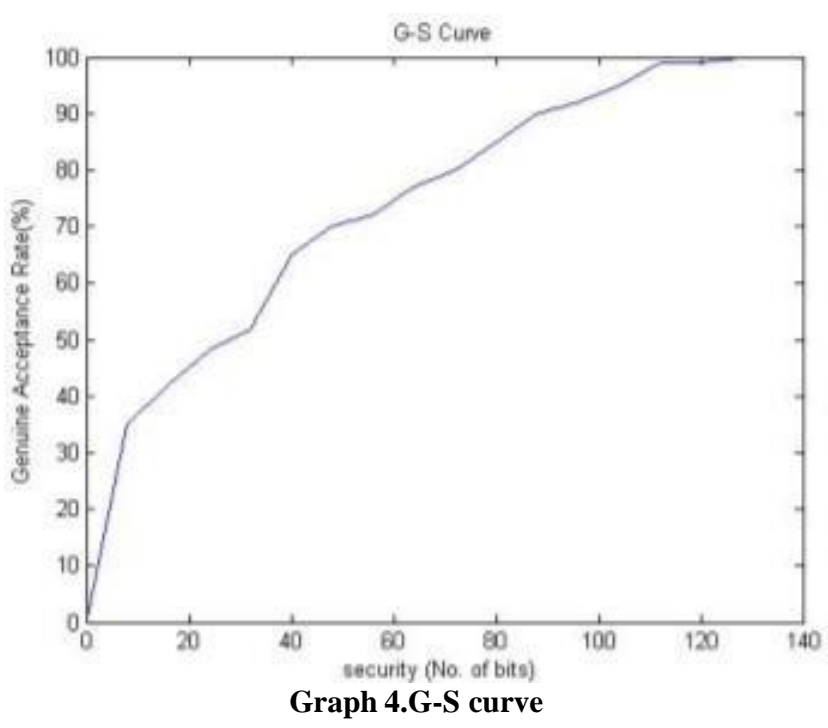

The third methodology is to check the False Rejection Rate (FRR) with GAR and False Acceptance Rate (FAR) is likewise thought of. The GAR, FAR, FRR are determined. In the event that the quantity of highlight focuses and fundamental focuses is high in unique finger impression and FKP, then, at that point the GAR is high and subsequently, the FRR turns out to below. An assortment of tests be cultivated to take a glance at the introduction of the extended methodology through the on top of components. The result procured from the proposed approach is $\mathrm{FRR}=0.6 \%, \mathrm{GAR}=99.4 \%$ and $\mathrm{FAR}=0 \%$.

Finally, a comparison of the planned approach with alternative approaches is done. Karthik et.al has proposed a theme for securing the multibiometric templates victimization fuzzy vault approach. Fingerprint and iris were investigated using MSU-DBI fingerprint and CASIA iris databases, achieving $\mathrm{GAR}=98.2 \%$ and $\mathrm{FAR}=0.01 \%$ with the protection of forty-one bits. Vincenzo Conti et al. have used frequency based mostly multibiometric systems with fingerprint and iris. They need to test their experiments using FVC2002 and tub for fingerprint and iris separately, getting the results of $\mathrm{GAR}=94.29 \%$ and $\mathrm{FRR}=5.71$. in line with this proposed approach, the GAR is expanded with more component focuses, central issues and with the most extreme group size. Analysis of the arranged methodology with the separate approach and its mythological huge curves. The general performance of this proposed approach is $\mathrm{GAR}=99.4 \%$ and $\mathrm{FRR}=0.6 \%$ with 128 bits security

VIII. COMPARATIVE STUDY OF BIOMETRIC APPROACHES

\begin{tabular}{|l|l|l|l|l|l|}
\hline \multicolumn{2}{|l|}{ Biometrics } & Mlgorithm & \multicolumn{2}{l}{ GAR (\%) } & FRR (\%) \\
\hline $\begin{array}{l}\text { Fingerprint } \\
\text { + IRIS }\end{array}$ & $\begin{array}{l}\text { Fuzzy vault based } \\
\text { approach Multimodal }\end{array}$ & $\begin{array}{l}\text { Biometric } \\
\text { system }\end{array}$ & 0.01 & 98.2 & 1.8 \\
\hline $\begin{array}{l}\text { Fingerprint } \\
+ \text { IRIS }\end{array}$ & $\begin{array}{l}\text { Frequency basedapproach } \\
\text { Multimodal }\end{array}$ & $\begin{array}{l}\text { biometric } \\
\text { system }\end{array}$ & 0 & 94.29 & 5.71 \\
\hline $\begin{array}{l}\text { Fingerprint } \\
\text { + FKP }\end{array}$ & $\begin{array}{l}\text { K-Means clustering Algorithm } \\
\text { (Proposed) }\end{array}$ & $\begin{array}{l}\text { Multimodal } \\
\text { Biometric } \\
\text { system }\end{array}$ & 0 & 99.4 & 0.6 \\
\hline
\end{tabular}

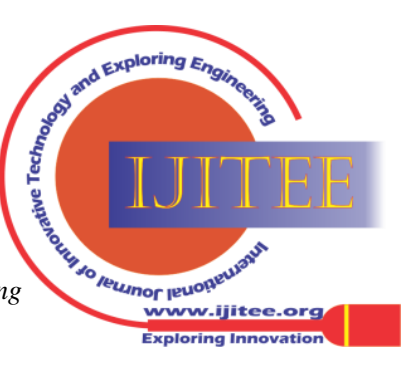




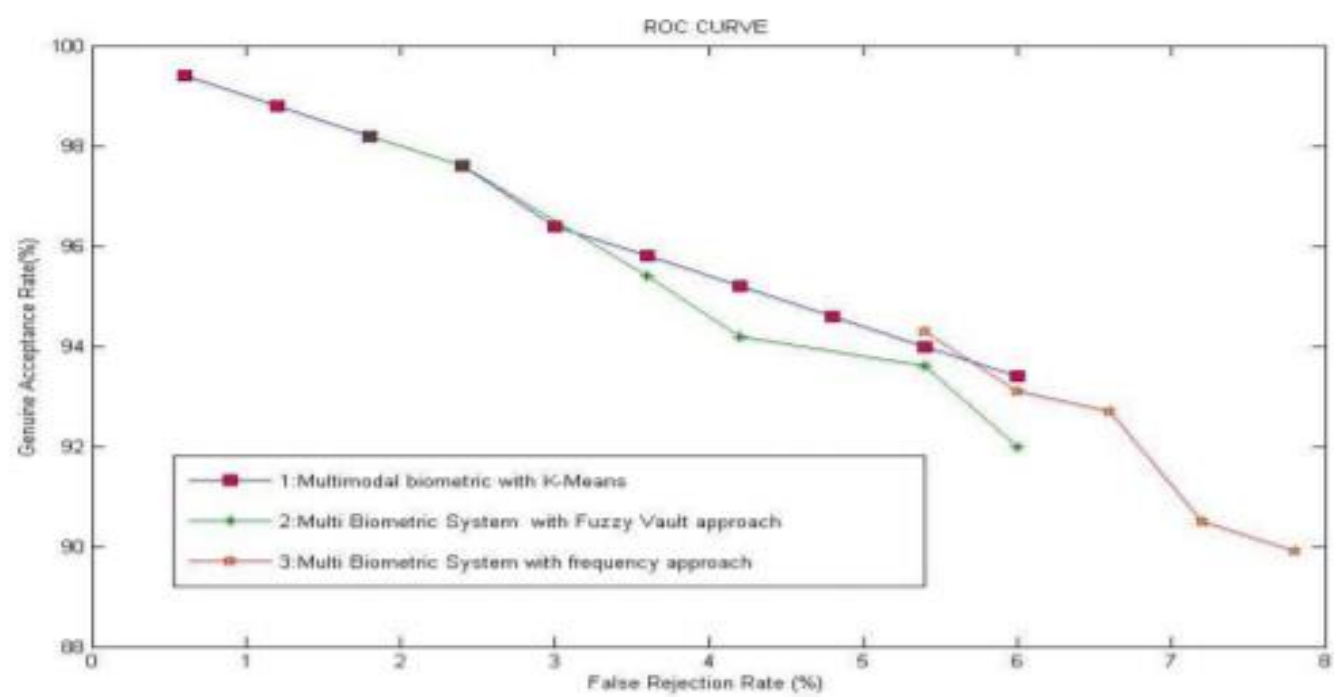

Graph 5.ROC arc of proposed the method contrast through accessible system

\section{RESULT}

A multimodal biometric system supported the fingerprint and FKP neglect K-Means cluster algorithm. For a perfect multibiometric system, the worth of GAR has got to be one hundred percent and FRR has to be nil. For several reasons like utilized images from the sensor, feature extraction method, matching process etc. $100 \%$ accuracy of recognition cannot be achieved. Therefore to enhance the acknowledgement accuracy, this paper proposes the generation of 128 bits binary values from fingerprint and FKP simultaneously. The two 128 bits are concatenated and hold on in the database. This fusion value of 256 bits are matched with a query input image with XOR operation then whether or not the user is real or fraud is decided. Supported this analysis work, the authentication is finished by the integration of the fingerprint and FKP with feature fusion. The planned multibiometric system provides authentication and security neglect K-Means cluster formula with GAR = $99.4 \%$, FRR $=0.6 \%$ and much $=0 \%$ with the security of 128 bits for every modality. These results are acquired by using the Poly U FKP information and FVC 2004 fingerprint database.

\section{CONCLUSION}

For an optimal verification framework, FAR and FRR records are equivalent to 0 . The previously mentioned result might be reached by online biometric verification frameworks, since they have the opportunity to dismiss the inferior quality obtained things. Conversely, officials prepared to utilize details (FVC data sets, CASIA, and so on) contain pictures with various quality, including low-, medium, and great biometric acquisitions, as limited and unethical pictures. Hence, these biometric confirmation frameworks don't accomplish the best outcome. To increment the connected security level, framework limitation are then set at that point to accomplish the FAR $=0 \%$ point and a relating FRR point.

\section{REFERENCES}

1. V. Bhawani Radhika, 'Biometric Identification Systems- Feature Level Clustering of Large Biometric Database,' NIT Rourkela,
May,2009.

2. Javad Khodadoust \&Ali Mohammad Khodadoust. Fingerprint indexing based on minutiae pairs and convex center point. Pattern Recognition, 2017.

3. NIST Fingerprint Image Quality. NIST Report, 2016.

4. Liu T, Zhu G, Zhang C, Vietnamese unit of activity P (2005) Fingerprint classification supported singular points. International Conference on Image Processing, pp 293-296 7.

5. Zhang, L., Zhang, L., Zhang, D. \& Zhu, H. (2011). Ensemble of native and international information for finger- knuckle-print recognition. Pattern Recognition, Vol. 44, No. 9, pp. 1990-1998.

6. A. MUTHUKUMAR AND S. KANNAN IJST, Transactions of Electrical Engineering, Vol. 37, No.E2, pp 133-145.

7. Anil, K. Jain, Ross, A. \& Pankanti, S. (2006). Biometrics: A tool for information security. IEEE Transactions on Information Forensics and Security, Vol. 1, No. 2, pp. 125-143. 2.

8. Kumar, A. \& Zhang, D. (2010). Improving biometric authentication performance from the user quality. IEEE Transactions on Instrumentation and Measurement, Vol. 59, No. 3, pp. 730-735. 3.

9. Kumar, A. \& Passi, A., (2010). Comparison and combination of iris matchers for reliable personal authentication. Pattern Recognition, Vol. 23, No. 3, pp. 1016-1026.

10. Kumar, A. \& Venkata Prathyusha, K. (2009). Personal authentication using hand vein triangulation and knuckle shape. IEEE Transactions on Image Processing, Vol. 18, No. 9, pp. $2127-$ 2136. 5.

11. Miguel, A. Ferrer, Carlos, M. Travieso \& Jesus, B. Alonso, (2006). Using hand knuckle texture for biometric identifications. IEEE Aerospace \& Electronics Systems Magazine, Vol. 43, No. 6, pp. 2327. 6.

12. Vincenzo Conti, Carmelo Militello, Filippo Sorbello, Member, IEEE, and Salvatore Vitabile, Member, IEEE. A Frequency Based Approach for features Fusion in Fingerprint and IRIS Multimodal Biometric Identification System.

13. Q. Zhao, F. Liu, and D. Zhang, 'A comparative study on quality assessment of high resolution fingerprint images,' in Image Processing (ICIP), 2010 17th IEEE International Conference on. IEEE, 2010, pp. 3089-3092.

14. A. Ross, K. Nandakumar, A.K. Jain 'Introduction to multibiometrics'Hand book of biometrics, Springer, Boston, MA (2008), pp. 271-292 Cross RefView Record in ScopusGoogle Scholar.

15. Rafael, C. Gonalez \& Richard, E. Woods, (2012). Digital image processing. Pearson Education, 3rd edition.

16. Poly U FKP, Database: http: // www. comp. polyu. edu. Hk / biometrics/FKP.htm.
Published By:

Blue Eyes Intelligence Engineering and Sciences Publication

(C) Copyright: All rights reserved.

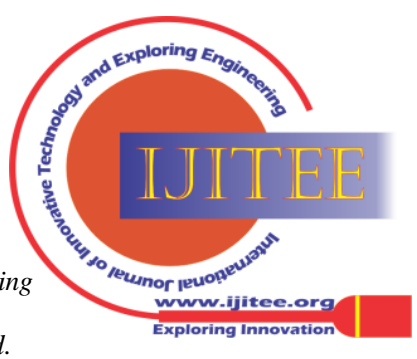




\section{AUTHORS PROFILE}

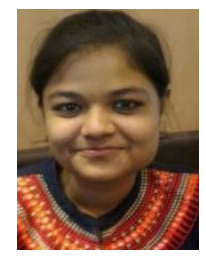

Varisha Alam, is pursuing M.Tech in Computer Science and Engineering. She is working with Ordering of Huge Biometric Information in Database System in fourth Semester MTech, in the Department of Computer Science and Engineering, Integral University, Lucknow, India. Her area of interests includes neural Network and fuzzy logics. Email Idvarisha626@gmail.com

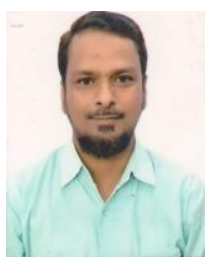

Dr. Mohammad Arif, received his B. Tech. in Computer Science \& Engineering from CCS University, Meerut, India. He perused his M. Tech in Computer Science \& Engineering from MN-NIT, Allahabad, India and Ph. D. in Computer Science \& Engineering from Integral University, Lucknow, India. He is working as Associate Professor in Department of Computer Science \& Engineering in Integral University, Lucknow, India. He is having nearly twenty years of teaching experience. He has got published nearly 40 peer reviewed papers in international journals and conferences. Email Id- arif.mohd2k@gmail.com 